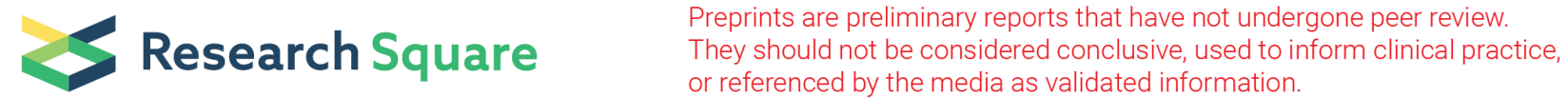

\section{Individualized Prediction of Stage I Colorectal Cancer Recurrence Using a Nomogram}

\author{
Aobo Zhuang \\ Zhongshan Hospital Fudan University \\ Dexiang Zhu \\ Zhongshan Hospital Fudan University \\ Qi Lin \\ Zhongshan Hospital Fudan University \\ Pingping $\mathrm{Xu}$ \\ Zhongshan Hospital Fudan University \\ Guodong He \\ Zhongshan Hospital Fudan University \\ Li Ren \\ Zhongshan Hospital Fudan University \\ Ye Wei \\ Zhongshan Hospital Fudan University \\ Jianmin Xu ( $\nabla$ xujmin@aliyun.com) \\ Zhongshan Hospital Fudan University
}

\section{Research Article}

Keywords: colorectal cancer, Zhongshan Hospital, Cox regression, helpfulhelpful in postoperative stage

Posted Date: May 7th, 2021

DOI: https://doi.org/10.21203/rs.3.rs-468523/v1

License: @) (1) This work is licensed under a Creative Commons Attribution 4.0 International License. Read Full License 


\section{Abstract \\ Background}

Though the prognosis of stage I colorectal cancer (CRC) is suitable, some patients still recurrence and have a poor prognosis. Few prognostic risk models have been proposed. Therefore, we aimed to identify factors affecting the recurrence in patients with stage I CRC and develop a predictive nomogram.

\section{Methods}

The nomogram was based on a retrospective study on patients who underwent radical surgery for stage I CRC at Zhongshan Hospital (Shanghai, China) between August 2008 and December 2016. Predictive factors for recurrence were determined and a nomogram predicting recurrence-free survival was constructed based on Cox regression. This model was internally validated, and performance was evaluated through calibration plots.

\section{Results}

A total of 1,359 patients who underwent curative surgery for stage I CRC were enrolled. With the 62.0 months median follow-up time,71 (5.2\%) experienced recurrence. The median time to recurrence was 24 months, $70 \%$ was diagnosed within three years after curative resection and $80 \%$ within 5 years. The 5 -year cumulative recurrence rate was $5.0 \%$, and the 10 -year recurrence rate was $6.6 \%$. In multivariate Cox analysis, age, preoperative serum CEA concentration, preoperative serum CA19-9 concentration, preoperative neutrophil-to-lymphocyte ratio, primary tumor location and lymphovascular invasion were the independent predictors of recurrence. A nomogram based on eight factors for recurrence-free survival was developed and internally validated. The concordance index of the nomogram was 0.716 .

\section{Conclusions}

For stage I CRC, more than one in every twenty people may experience recurrence within 10 years after radical surgery. The nomogram we developed and internally validated might be helpfulhelpful in postoperative stage I CRC surveillance.

\section{Introduction}

Colorectal cancer(CRC) is the third most prevalent malignancy and a leading cause of cancer-related death worldwide[1]. The 5-years overall survival (OS) rate of patients with $\mathrm{CRC}$ has increased markedly. These improvements are not only due to advances in treatment

strategies but to early detection but owing to cancer screening programs [2]and the popularization of digestive endoscopy [3, 4]. As screening colonoscopy rates have increased, so has the proportion of patients diagnosed with stage I CRC.[5]. It has been thought that stage I CRC(pT1-2N0M0) has an excellent prognosis after radical resection with a 5 -year OS rate of more than $90 \%$ [6], and some even are cured. The standard surveillance schedule after curative resection for colorectal cancer in Japan comprises serum tumor marker measurements every 3 months for the first 3 years and every 6 months for the next 2 years, computed tomography (CT) for chest and abdomen every 6 months for the first 3 years and every 12 months for the next 2 years, and colonoscopy every 1-2 years. This schedule seems to be rather intensive for stage I CRC. There only have about 4.1 to $9.8 \%$ stage I CRC recurrence[7-9].]. Due to its relatively low recurrence rate, few nomograms have been established. Therefore, our study aimed to identify clinicopathologic factors related to recurrence and to develop a predictive nomogram, which could accurately predict the probability of recurrence in stage I colorectal cancer.probability of recurrence in stage I colorectal cancer.

\section{Materials And Methods}

Patients and enrollment

This study was a retrospective design based on a prospectively collected institutional database at the General Surgery Department, Zhongshan Hospital, Fudan University, Shanghai, China. Between August 2008 and December 2016, patients with stage I CRC(the description was based on the 8th edition of the American Joint Committee on Cancer grading system) who underwent curative surgical resection were included. Patients with multiple primary CRC or with a history of other malignancies were excluded. Patients who had chemoradiotherapy before surgery or patients with follow-up less than one year after surgery were also excluded. This study was approved by the institutional review board of Zhongshan Hospital, Fudan University (Shanghai, China), and was carried out following the Declaration of Helsinki. All patients provided written informed consent.

Clinicopathologic evaluation

Clinicopathologic variables that potentially predict tumor recurrence were collected, including age, sex, preoperative serological indicators, tumor location, tumor differentiation, tumor size, lymphovascular invasion, number of retrieved lymph nodes, depth of tumor penetration into the colon wall ( $T$ stage) and surgical approach. According to the distance from the anal verge(AV) to the lower border of the tumor (per rigid scope: 0 to $5 \mathrm{~cm}$ and $5 \mathrm{~cm}$ to $15 \mathrm{~cm}$ ) [10], we divided the rectal tumors into two groups for statistical analysis: Middle-high rectal cancer(HR) and lower rectal cancer(LR). Besides, right-sided colon cancer (RC) is the tumor in the cecum, ascending colon, hepatic flexure, or the proximal two-thirds of transverse colon, left-sided colon cancer (LC) is the tumor arising in the splenic flexure, descending colon, sigmoid colon, or rectosigmoid colon. We took blood before surgery and obtained the neutrophil-to-lymphocyte ratio (NLR) by dividing neutrophil count by lymphocyte count. 
The follow-up schedule was based on the Chinese guidelines for colorectal cancer[11]. Patients were routinely followed every 3 months after surgery for the first 2 years, every 6 months for another 3 years and annually thereafter. The evaluation included a clinical examination, measurement of the levels of tumour markers including carcinoembryonic antigen (CEA) and CA19-9 and CT scans of the chest, abdomen and pelvis. Colonoscopy was performed every 6 months. If the patient was suspected of recurrence, he/she would do a further examination, including biopsy, colonoscopy and tumor-related imaging examination. The diagnosis of lung metastasis is made by pathological diagnosis. If the pathological diagnosis cannot be achieved, enhanced CT and PET-CT were be used[12]. Liver metastasis is diagnosed based on Chinese guidelines for colorectal liver metastases[13]. Local recurrence (LR) is the tumor recurrence confined to the primary tumor's regional area, including recurrence of pericolic fat, mesentery, lymph nodes, or intraluminal recurrence.

\section{Statistical analysis}

Recurrence-free survival(RFS) was calculated in months from the date of radical surgery to the time of detection of tumor recurrence, death, last date of follow-up. Clinicopathologic variables such as sex, high-level preoperative serum CEA or CA19-9 concentration, the incidence of decreased level of prealbumin or albumin, surgical approach, tumor location, LVI and T category were compared using Fisher's exact test or the Pearson Chi-square between recurrent and non-recurrent. Age retrieved lymph nodes and tumor size were compared by using an independent samples t-test. We calculated and compared the survival rate by using the Kaplan-Meier method, the log-rank test. The Cox regression model was used to assess the independent factors of tumor recurrence. A nomogram for predicting stage I CRC was created with the statistically significant variables and evaluated using the concordance index (c index) and calibration plots. The specific scores of each factor in the nomogram are calculated using the coefficients from cox regression. A point system of nomogram is used to assign each predictor, with point ranges from 0 to 100 in a graphic interface. Based on the estimated regression coefficients, we rank the estimated effects, disregarding statistical significance and direction (absolute beta values). We determined which predictor has the most significant impact in the model, then sequentially assigned other predictors based on their proportions to the point assigned to the most prominent impact predictor. The presence of other predictors influences each predictor. Finally, we used estimated beta coefficients from a primary effect cox model. All tests were two-tailed and statistical significance was $\mathrm{P}<0.05$. All data were analyzed using SPSS 22.0 (SPSS Inc., Chicago, IL, USA) and R 3.1.1 (R Foundation for Statistical Computing, Vienna, Austria; http://www.r-project.org/).

\section{Results}

Between August 2008 and December 2016, 1533 patients were diagnosed with stage I CRC at the General Surgery Department, Zhongshan Hospital, Fudan University, Shanghai, China. After removing patients with hereditary $\operatorname{CRC}(n=38)$, patients with a history of other malignancies ( $n=59)$, patients who had undergone chemoradiotherapy before surgery $(n=35)$, and patients with follow-up less than one year after surgery $(n=42), 1359(88.6 \%)$ patients were chosen for analysis. (Fig. 1) The median follow-up time was 62.0 months for those patients, and 554 (40.8\%) had colon cancer; $805(59.2 \%)$ had rectal cancer. Most of them were diagnosed tumors classified as $\mathrm{T} 2(\mathrm{~N}=815 ; 60.1 \%)$. Recurrence was significantly related to old age $(\mathrm{p}=0.004)$, higher preoperative serum $\mathrm{CEA}$ concentration $(p=0.014)$, higher preoperative serum CA19-9concentration $(p=0.003)$, higher NLR(0.008), tumor location $(p=0.014)$, differentiation $(0.028), L V I$ $(p=0.008)$ and T category $(p=0.004)($ Table 1$)$. 
Table 1

Demographic and pathologic characteristics according to cancer recurrence

\begin{tabular}{|c|c|c|c|c|}
\hline \multirow[t]{2}{*}{ Variables } & \multicolumn{3}{|c|}{ Values as mean \pm SD or no. of patients (\%) } & \multirow[t]{2}{*}{ P value } \\
\hline & Total $(n=1359)$ & Non-Recurrence $(n=1288)$ & Non-recurrence $(\mathrm{n}=71)$ & \\
\hline Age, years & $62.8(51.6-64.0)$ & $62.6(51.4-73.7)$ & $66.5(55.4-77.5)$ & 0.004 \\
\hline Sex & $761(56.0)$ & $718(55.7)$ & $43(60.6)$ & 0.426 \\
\hline Male & $598(44.0)$ & $570(44.3)$ & 28(39.4) & \\
\hline \multicolumn{5}{|l|}{ Female } \\
\hline NLR & 282(20.8) & $257(20.0)$ & 25(35.2) & 0.008 \\
\hline$>=2.5$ & $985(72.5)$ & $942(73.1)$ & $43(60.6)$ & \\
\hline$\otimes 2.5$ & $92(6.8)$ & $89(6.9)$ & $3(4.2)$ & \\
\hline \multicolumn{5}{|l|}{ Unknown } \\
\hline Prealbumin,g/L & $584(43.0)$ & $545(42.3)$ & $39(54.9)$ & 0.097 \\
\hline$<0.25$ & $673(49.5)$ & $645(50.1)$ & $27(38.0)$ & \\
\hline$>=0.25$ & $94(6.9)$ & $89(6.9)$ & $5(7.0)$ & \\
\hline \multicolumn{5}{|l|}{ Unknown } \\
\hline Albumin,g/L & $86(6.3)$ & $78(6.1)$ & $8(11.3)$ & 0.214 \\
\hline$<35$ & 1212(89.2) & 1152(89.4) & $60(84.5)$ & \\
\hline$>=35$ & $61(4.5)$ & $58(4.5)$ & $3(4.2)$ & \\
\hline \multicolumn{5}{|l|}{ Unknown } \\
\hline $\mathrm{CEA}, \mathrm{ng} / \mathrm{mL}$ & 188(13.8) & $170(13.2)$ & $18(25.4)$ & 0.014 \\
\hline$>=5$ & 1102(81.1) & 1053(81.8) & $49(69.0)$ & \\
\hline$<5$ & $69(5.1)$ & $65(5.0)$ & $4(5.6)$ & \\
\hline \multicolumn{5}{|l|}{ Unknown } \\
\hline CA19-9,u凹ml & $34(2.5)$ & $28(2.2)$ & $6(8.5)$ & 0.003 \\
\hline$>=37$ & 1239(91.2) & 1180(91.6) & $59(83.1)$ & \\
\hline$<37$ & $86(6.3)$ & $80(6.2)$ & $6(8.5)$ & \\
\hline \multicolumn{5}{|l|}{ Unknown } \\
\hline Tumor location & 192(14.1) & $186(14.4)$ & $6(8.5)$ & 0.014 \\
\hline Right colon & $274(20.2)$ & $256(19.9)$ & $18(25.4)$ & \\
\hline Left colon & $587(43.2)$ & $565(43.9)$ & $22(31.0)$ & \\
\hline Middle-High rectal & $306(22.5)$ & $281(21.8)$ & $25(35.2)$ & \\
\hline \multicolumn{5}{|l|}{ Lower rectal } \\
\hline Tumor size, $\mathrm{cm}$ & $3.0(1.6-4.5)$ & $3.0(1.6-4.5)$ & $3.1(1.9-4.3)$ & 0.626 \\
\hline Differentiation & $978(72.0)$ & $935(72.6)$ & $43(60.6)$ & 0.028 \\
\hline WD/MD & $381(28.0)$ & $353(27.4)$ & $28(39.4)$ & \\
\hline \multicolumn{5}{|l|}{$\mathrm{PD} / \mathrm{MUC}$} \\
\hline LVI & $101(7.4)$ & $90(7.0)$ & $11(15.5)$ & 0.008 \\
\hline Positive & 1258(92.6) & 1198(93.0) & $60(84.5)$ & \\
\hline \multicolumn{5}{|l|}{ Negative } \\
\hline T stage & $544(40.0)$ & $527(40.9)$ & 17(23.9) & 0.004 \\
\hline $\mathrm{T} 1$ & $815(60.0)$ & 761(59.1) & $54(76.1)$ & \\
\hline \multicolumn{5}{|l|}{$\mathrm{T} 2$} \\
\hline Retrieved LN & $13.4(6.3-20.4)$ & $13.4(6.5-20.3)$ & $12.4(3.4-21.3)$ & 0.215 \\
\hline
\end{tabular}




\begin{tabular}{|lllll|}
\hline Variables & \multicolumn{2}{l}{ Values as mean \pm SD or no. of patients (\%) } & P value \\
\hline Surgical approach & $690(50.8)$ & $658(51.1)$ & $32(45.1)$ & 0.324 \\
Laparoscope & $669(49.2)$ & $630(48.9)$ & $39(54.9)$ & \\
Open & & & & \\
\hline
\end{tabular}

Recurrence pattern

In 1359 patients,71 (5.2\%) experienced recurrence, the median recurrence time was 24 months (IQR 14-43 months), 69\%(69(n= 50) was diagnosed within three years after CRC diagnosis, $899 \%(n=63)$ within 5 years. The 5 -year cumulative recurrence rate was $5.0 \%$, and the 10 -year recurrence rate was $6.6 \%$.

For the recurrence patients, $63(88.7 \%)$ with single metastases and $8(11.3 \%)$ with multiple metastases. Lung is the most common site of metastasis $(\mathrm{N}=32$; $45.1 \%)$, followed by liver $(\mathrm{N}=23 ; 32.4 \%)$, than local recurrence $(\mathrm{N}=14 ; 19.7 \%)$. For the lung recurrence, the median recurrence time was 27 months (IQR $14-35$ months), the 5 -year cumulative recurrence rate was $1.6 \%$, and the 10 -year recurrence rate was $2.3 \%$. For the liver recurrence, the median recurrence time was 23 months (IQR 7-37 months), the 5-year cumulative recurrence rate was 1.3\%, and the 10-year recurrence rate was 1.8\%; For the local recurrence, the median recurrence time was 22 months (IQR 18-43 months), the 5-year cumulative recurrence rate was 1.0\%, and the 10-year recurrence rate was $1.0 \%$.

Using a log-rank testtest for RFS, we found that: compared to other primary sites, LR was more likely to have lung metastases; compared to RC and HR, LC and LR were more likely to have liver metastases; rectal cancers were more likely to have a local recurrence than colon cancer (Fig. 2 ).

Factors Associated With RFS

Univariate analyses

From the univariate analysis, we found that old age, higher preoperative serum CEA concentration, higher preoperative serum CA19-9 concentration, higher Preoperative NLR, tumor location, differentiation, T category and LVI were associated with low RFS rates $(P<0.05)(T a b l e ~ 2)$. 
Table 2

Cox regression analysis of recurrence-free survival in all study patients

\begin{tabular}{|c|c|c|c|}
\hline \multirow[t]{2}{*}{ Variables } & \multirow{2}{*}{$\begin{array}{l}\text { Univariate analysis } \\
\text { P value }\end{array}$} & \multicolumn{2}{|c|}{ Multivariate analysis } \\
\hline & & $P$ value & $\mathrm{HR}(\mathrm{Cl})$ \\
\hline Age & 0.005 & 0.011 & $1.029(1.007-1.052)$ \\
\hline Sex & 0.399 & & \\
\hline \multicolumn{4}{|l|}{ Male } \\
\hline \multicolumn{4}{|l|}{ Female } \\
\hline NLR & 0.004 & 0.004 & $2.087(1.263-3.448)$ \\
\hline$>=2.5$ & & & 1 \\
\hline \multicolumn{4}{|l|}{$\bigotimes 2.5$} \\
\hline Prealbumin & 0.099 & 0.142 & \\
\hline \multirow{2}{*}{\multicolumn{4}{|c|}{$\begin{array}{l}<0.25 \\
>=0.25\end{array}$}} \\
\hline & & & \\
\hline Albumin & 0.176 & & \\
\hline \multicolumn{4}{|l|}{$<35$} \\
\hline \multicolumn{4}{|l|}{$>=35$} \\
\hline CEA & 0.002 & 0.042 & $1.802(1.021-3.185)$ \\
\hline$>=5$ & & & 1 \\
\hline \multicolumn{4}{|l|}{$<5$} \\
\hline CA19-9 & 0.001 & 0.003 & $3.717(1.560-8.850)$ \\
\hline$>=37$ & & & 1 \\
\hline \multicolumn{4}{|l|}{$<37$} \\
\hline Tumor location & 0.009 & 0.011 & 1 \\
\hline Right colon & & & $2.583(1.012-6.591)$ \\
\hline Left colon & & & $1.147(0.457-2.876)$ \\
\hline Middle-High rectal & & & $2.499(1.011-6.179)$ \\
\hline \multicolumn{4}{|l|}{ Lower rectal } \\
\hline Tumor size & 0.558 & & \\
\hline Differentiation & 0.009 & 0.103 & \\
\hline \multicolumn{4}{|l|}{ WD/MD } \\
\hline \multicolumn{4}{|l|}{$\mathrm{PD} / \mathrm{MUC}$} \\
\hline LVI & 0.002 & 0.001 & $3.020(1.551-5.877)$ \\
\hline Positive & & & 1 \\
\hline \multicolumn{4}{|l|}{ Negative } \\
\hline T stage & 0.006 & 0.150 & \\
\hline \multicolumn{4}{|l|}{ T1 } \\
\hline \multicolumn{4}{|l|}{ T2 } \\
\hline Retrieved LN & 0.541 & & \\
\hline Surgical approach & 0.909 & & \\
\hline Laparoscope & & & \\
\hline Open & & & \\
\hline
\end{tabular}

Multivariate analyses

We used a cox regression model to assess the independent factors affecting RFS. Old age, higher preoperative serum CEA concentration, higher preoperative serum CA19-9 concentration, high preoperative NLR, tumor location and LVI were the independent factors of tumor recurrence in the proportional hazards 
model analysis $(P<0.05)$. However, there was no statistically significant difference relating to differentiation and $T$ category $(P>0.05)($ Table 2$)$.

Nomogram Construction and Internal Validation

Based on stage I CRC patients' risk factors, we developed a nomogram to predict 1-, 3-,5-, and 10-year recurrence(Fig. 3A). We added the eight risk factors item by item to obtained total points on the nomogram. Then we got the ratio of 1-,3-, 5-, or 10-year RFS by drawing a vertical line from the "total points" axis to the "recurrence-free probability" axis. In this nomogram, the increase of total points means the increase of recurrence. With a concordance index of 0.716 , the nomogram can accurately predict individual RFS.

Moreover, the calibration plot of the validation group showed good calibration(Fig. 3B). Furthermore, we stratified patients into 3 subgroups according to the nomogram-predicted score and compared the RFS curves within each subgroup. As shown in Figs. 4 the prognoses were almost identical between the cohort in each subgroup.

\section{Discussion}

Because of the national screening strategy and the popularity of endoscopy, more and more colorectal cancers are diagnosed early [4]. Due to its relatively low recurrence rate, few studies try to characterize stage I CRC's recurrence pattern after surgery. On the other hand, many nomograms designed to predict colorectal cancer prognosis have been developed at an accelerated pace[14-17], possibly owing to their high utility in daily clinical practice. Inin this retrospective study, we developed a nomogram for accurate prediction of recurrence in stage I colorectal cancer. With this nomogram, the postoperative recurrence rate can be calculated quantitatively based on the patient's clinicopathologic factors at the time of surgery. Thus, it isthus possible to identify patients with a high risk of recurrence after surgery and conduct a more active surveillance program to detect the recurrence as early as possible.

Our study median time of recurrence was 26 months, which is longer than in previous studies[8, 18-23]. More rectal cancer may lead to more lung metastasis. In general, the LR veins and lymphatics are drained to the common iliac vein and lymphatics, then to the systemic circulation, while the veins and lymphatics of the colon and HR are drained to the mesenteric system and then to the portal system[24, 25]. Mesenteric lymphatic vessels and blood vessels are removed during radical surgery but not in the iliac system[26]. Therefore, occult spreading to the iliac region may lead to the recurrence of LR cancer.

Furthermore, compared to upper rectal and colon cancer patients, we observed a higher lung metastases rate among lower rectal cancer patients [27, 28]. A previous study exploring CRC patients who experienced curative resection illustrated that lung metastasis tends to have a more prolonged RFS than liver metastasis (46 months vs 28 months) [29]. This information should be considered in determining an optimal follow-up scheme.

In this study, six variables were found to be pivotal factors determining recurrence. In our study, older patients had shorter survival times than their younger counterparts. The prognostic impact of age remains controversial; some studies found that younger age was a poor prognostic factor[30]. That's may because younger patients had more locally advanced and less favourable differentiation than older patients when cancer was diagnosed[31, 32]. When controlling for other clinicopathologic characteristics, young patients had a superior prognosis[33].

Preoperative serum CEA and CA19-9 were found to correlate with recurrence after radical surgery of stage I colorectal cancer. CEA is a complex glycoprotein used as an inter-cellular adhesion molecule[34]. Functioned as an adhesion molecule, CA19-9 is anticipated in tumor progression[35]. Our study firstly reports the predictive value of CA19-9 in stage I CRC. CA 19-9 has been widely used in diagnosing and prognosis of pancreatic cancer, gastric cancer, and other gastrointestinal tumors [36-38]. However,, the function of CA 19-9 in CRC remains controversial due to its relatively low sensitivity [39]. The presence of CA 19-9 correlates with tumor cell-induced platelet aggregation, which is involved in the distant metastasis of CRC[40].

In stage I tumors undergoing curative operations, NLR as a predictor of recurrence, thus may be a novel biomarker for tailored therapy. One meta-analysis of 66 articles recently indicated that high preoperative NLR values negatively impact cancer patients' RFS [41]. Some studies focused on the relationship between early-stage colon cancers and NLR prognosis, but none of these studies included patients with stage I patients alone[42-44].

The primary tumor site is also an independent risk factor for recurrence of stage I colorectal cancer after surgery. The poorer prognosis observed in LR patients may be determined by physiological anatomy. Generally speaking, the LR veins and lymphatics are drained to the common iliac vein and lymphatics, then to the systemic circulation, while the veins and lymphatics of the colon and HR are drained to the mesenteric system and then to the portal system[24, 25]. Mesenteric lymphatic vessels and blood vessels are removed during radical surgery but not in the iliac system[26]. Therefore, occult spreading to the iliac region may lead to the recurrence of LR cancer. Furthermore, compared to upper rectal and colon cancer patients, we observed a higher lung metastases rate among lower rectal cancer patients $[27,28]$. This information should be considered in determining an optimal follow-up scheme.

Our results indicate that lymphovascular permeation has been linked with poor prognosis. Lymphovascular invasion can occur intramurally within the colorectal wall itself or in the surrounding tissue. linuma et al.[45] found venous invasions significantly correlated with the presence of isolated tumor cells in blood samples. Furthermore, another study reported that detecting circulating tumor cells in the peripheral blood helped determine the patients at high risk for recurrence[46].

In this study, we developed a nomogram based on clinical-pathological factors and serological indicators. By using it, we calculated the postoperative recurrence rate based on clinicopathologic factors. This nomogram also could identify patients at high risk of recurrence after surgery and conduct a supervision program to detect the recurrence early. Removing metastases and good treating could free prolonged disease survival for patients whose recurrence was found early. 
There are several limitations of this study. Firstly, it's a retrospective study without external validation. Secondly, a few patients were excluded due to incomplete data. Thirdly, results of PNI, IHC staining of p53 and MMR gene status were not evaluated for the entire study period because they were not regularly determined before 2012 , so we can't directly compare our findings with molecular research.

In conclusion, more than one in every twenty people may experience recurrence within ten years after radical surgery. We developed a simple and accurate nomogram that predicts the recurrence of stage I CRC. This nomogram may help physicians identify high-risk patients who need more active surveillance and ensure efficient disease management.

\section{Declarations}

Ethics approval and consent to participate

This study was approved by the institutional review board of Zhongshan Hospital, Fudan University (Shanghai, China), and was carried out in accordance with the Declaration of Helsinki. All patients provided written and oral informed consent.

Consent for publication

All patients provided written and oral informed consent.

Availability of data and materials

The datasets used and analysed during the current study are available from the corresponding author on reasonable request.

Competing interests

No potential conflicts of interest were disclosed.

Funding

There is no funding for this study.

Authors' contributions

ZAB, ZDX and LQ carried out the concepts, design, definition of intellectual content, literature search, data acquisition, data analysis and manuscript preparatio

Acknowledgements

Not applicable

\section{References}

[1]. Siegel, R.L., K.D. Miller and A. Jemal, Cancer Statistics, 2017. CA Cancer J Clin, 2017. 67(1): p. 7-30.

[2]. Morris, E.J.A., et al., A retrospective observational study of the relationship between family history and survival from colorectal cancer. British Journal of Cancer, 2013. 108(7): p. 1502-1507.

[3]. Amri, R., et al., Impact of Screening Colonoscopy on Outcomes in Colon Cancer Surgery. JAMA Surgery, 2013. $148(8)$ : p. 747.

[4]. Zorzi, M., et al., Screening for colorectal cancer in Italy: 2011-2012 survey. Epidemiol Prev, 2015. 39(3 Suppl 1): p. $115-25$.

[5]. Zorzi, M., et al., Screening for colorectal cancer in Italy: 2011-2012 survey. Epidemiol Prev, 2015. 39(3 Suppl 1): p. 93-107.

[6]. Gonzalez-Pons, M. and M. Cruz-Correa, Colorectal Cancer Biomarkers: Where Are We Now? BioMed Research International, 2015. 2015 : p. 1-14.

[7]. Wichmann, M.W., et al., Results of long-term follow-up after curative resection of Dukes A colorectal cancer. World J Surg, 2002. 26(6): p. 732-6.

[8]. Lee, J.H., et al., Identification of Recurrence-Predictive Indicators in Stage I Colorectal Cancer. World Journal of Surgery, 2017. 41(4): p. 1126-1133.

[9]. Kobayashi, H., et al., Characteristics of recurrence and surveillance tools after curative resection for colorectal cancer: a multicenter study. Surgery, 2007. 141(1): p. 67-75.

[10]. Kusters, M., et al., Patterns of local recurrence in rectal cancer; a study of the Dutch TME trial. Eur J Surg Oncol, 2010. 36(5): p. 470-6.

[11]. [Chinese Protocol of Diagnosis and Treatment of Colorectal Cancer]. Zhonghua Wai Ke Za Zhi, 2018. 56(4): p. $241-258$.

[12]. Li, J., et al., Expert consensus on multidisciplinary therapy of colorectal cancer with lung metastases (2019 edition). J Hematol Oncol, 2019. 12(1): p. 16. 
[13]. Xu, J., et al., Chinese guidelines for the diagnosis and comprehensive treatment of colorectal liver metastases (version 2018). J Cancer Res Clin Oncol, 2019. 145(3): p. 725-736.

[14]. Weiser, M.R., et al., Individualized Prediction of Colon Cancer Recurrence Using a Nomogram. Journal of Clinical Oncology, 2008. 26(3

): p. 380-385.

[15]. Kawai, K., et al., Nomogram Predicting Survival After Recurrence in Patients With Stage I to III Colon Cancer. Diseases of the Colon \& Rectum, 2018. 61(9): p. 1053-1062.

[16]. Kawai, K., et al., Nomogram Prediction of Metachronous Colorectal Neoplasms in Patients With Colorectal Cancer. Annals of Surgery, 2015. 261(5): p. 926-932.

[17]. Kim, C., et al., Predictive Nomogram for Recurrence of Stage I Colorectal Cancer After Curative Resection. Clinical Colorectal Cancer, 2018. 17(3): p. e513-e518.

[18]. Xu, B., et al., Prognostic factors in the patients with T2NOMO colorectal cancer. World Journal of Surgical Oncology, 2016. 14(1).

[19]. Teloken, P.E., et al., Recurrence in patients with stage I colorectal cancer. ANZ J Surg, 2016. 86(1-2): p. 49-53.

[20]. Lee, J.H., et al., Identification of Recurrence-Predictive Indicators in Stage I Colorectal Cancer. World Journal of Surgery, 2017. 41(4): p. 1126-1133.

[21]. Chok, K.S.H. and W.L. Law, Prognostic Factors Affecting Survival and Recurrence of Patients with pT1 and pT2 Colorectal Cancer. World Journal of Surgery, 2007. 31(7): p. 1485-1490.

[22]. Losi, L., et al., Prognostic significance of histological features and biological parameters in stage I (pT1 and pT2) colorectal adenocarcinoma. Pathology - Research and Practice, 2006. 202(9): p. 663-670.

[23]. Cihan, S., et al., Recurrence Risk and Prognostic Parameters in Stage I Rectal Cancers. Asian Pacific Journal of Cancer Prevention, 2014. 15(13): p. 5337-5341.

[24]. Watanabe, K., et al., Predictive factors for pulmonary metastases after curative resection of rectal cancer without preoperative chemoradiotherapy. Dis Colon Rectum, 2011. 54(8): p. 989-98.

[25]. Lee, J.L., et al., Rate of pulmonary metastasis varies with location of rectal cancer in the patients undergoing curative resection. World J Surg, 2015. 39(3): p. 759-68.

[26]. Willett, C.G., et al., Are there patients with stage I rectal carcinoma at risk for failure after abdominoperineal resection? Cancer, 1992. 69(7): p. 1651-5.

[27]. van Gestel, Y.R., et al., Metachronous peritoneal carcinomatosis after curative treatment of colorectal cancer. Eur J Surg Oncol, 2014. 40(8): p. 963-9.

[28]. Kobayashi, H., et al., Characteristics of recurrence and surveillance tools after curative resection for colorectal cancer: a multicenter study. Surgery, 2007. 141(1): p. 67-75.

[29]. Sadahiro, S., et al., Recurrence patterns after curative resection of colorectal cancer in patients followed for a minimum of ten years. Hepatogastroenterology, 2003. 50(53): p. 1362-6.

[30]. Palmer, M.L., L. Herrera and N.J. Petrelli, Colorectal adenocarcinoma in patients less than 40 years of age. Dis Colon Rectum, 1991. 34(4): p. 343-6.

[31]. O'Connell, J.B., et al., Are survival rates different for young and older patients with rectal cancer? Dis Colon Rectum, 2004. 47(12): p. 2064-9.

[32]. Derwinger, K., K. Kodeda and R. Gerjy, Age aspects of demography, pathology and survival assessment in colorectal cancer. Anticancer Res, 2010. 30(12): p. 5227-31.

[33]. McKay, A., et al., Does young age influence the prognosis of colorectal cancer: a population-based analysis. World J Surg Oncol, 2014. 12: p. 370.

[34]. Benchimol, S., et al., Carcinoembryonic antigen, a human tumor marker, functions as an intercellular adhesion molecule. Cell, 1989. 57(2): p. 327-34.

[35]. Del, V.B., et al., Radioimmunometric assay for a monoclonal antibody-defined tumor marker, CA 19-9. Clin Chem, 1983. 29 (3): p. 549-52.

[36]. Chen, Y., et al., Serum CA242, CA199, CA125, CEA, and TSGF are Biomarkers for the Efficacy and Prognosis of Cryoablation in Pancreatic Cancer Patients. Cell Biochem Biophys, 2015. 71(3): p. 1287-91.

[37]. Wang, Q., et al., Prognostic value of carbohydrate tumor markers and inflammation-based markers in metastatic or recurrent gastric cancer. Med Oncol, 2014. 31(12): p. 289.

[38]. Polat, E., et al., Diagnostic value of preoperative serum carcinoembryonic antigen and carbohydrate antigen 19-9 in colorectal cancer. Curr Oncol, 2014. 21(1): p. e1-7. 
[39]. Galli, C., D. Basso and M. Plebani, CA 19-9: handle with care. Clin Chem Lab Med, 2013. 51(7): p. 1369-83.

[40]. Martini, F., et al., CA 19-9 monosialoganglioside content of human colorectal tumor cells correlates with tumor cell-induced platelet aggregation. Anticancer Res, 2000. 20(3A): p. 1609-14.

[41]. Mei, Z., et al., Prognostic role of pretreatment blood neutrophil-to-lymphocyte ratio in advanced cancer survivors: A systematic review and metaanalysis of 66 cohort studies. Cancer Treat Rev, 2017. 58: p. 1-13.

[42]. Galizia, G., et al., Neutrophil to lymphocyte ratio is a strong predictor of tumor recurrence in early colon cancers: A propensity score-matched analysis. Surgery, 2015. 158(1): p. 112-120.

[43]. Oh, S.Y., Y.B. Kim and K.W. Suh, Prognostic significance of systemic inflammatory response in stage II colorectal cancer. Journal of Surgical Research, 2017. 208: p. 158-165.

[44]. Ding, P., et al., Elevated preoperative neutrophil to lymphocyte ratio predicts risk of recurrence following curative resection for stage IIA colon cancer. International Journal of Colorectal Disease, 2010. 25(12): p. 1427-1433.

[45]. linuma, H., et al., Clinical significance of circulating tumor cells, including cancer stem-like cells, in peripheral blood for recurrence and prognosis in patients with Dukes' stage B and C colorectal cancer. J Clin Oncol, 2011. 29(12): p. 1547-55.

[46]. linuma, H., et al., Usefulness and clinical significance of quantitative real-time RT-PCR to detect isolated tumor cells in the peripheral blood and tumor drainage blood of patients with colorectal cancer. Int J Oncol, 2006. 28(2): p. 297-306.

\section{Figures}

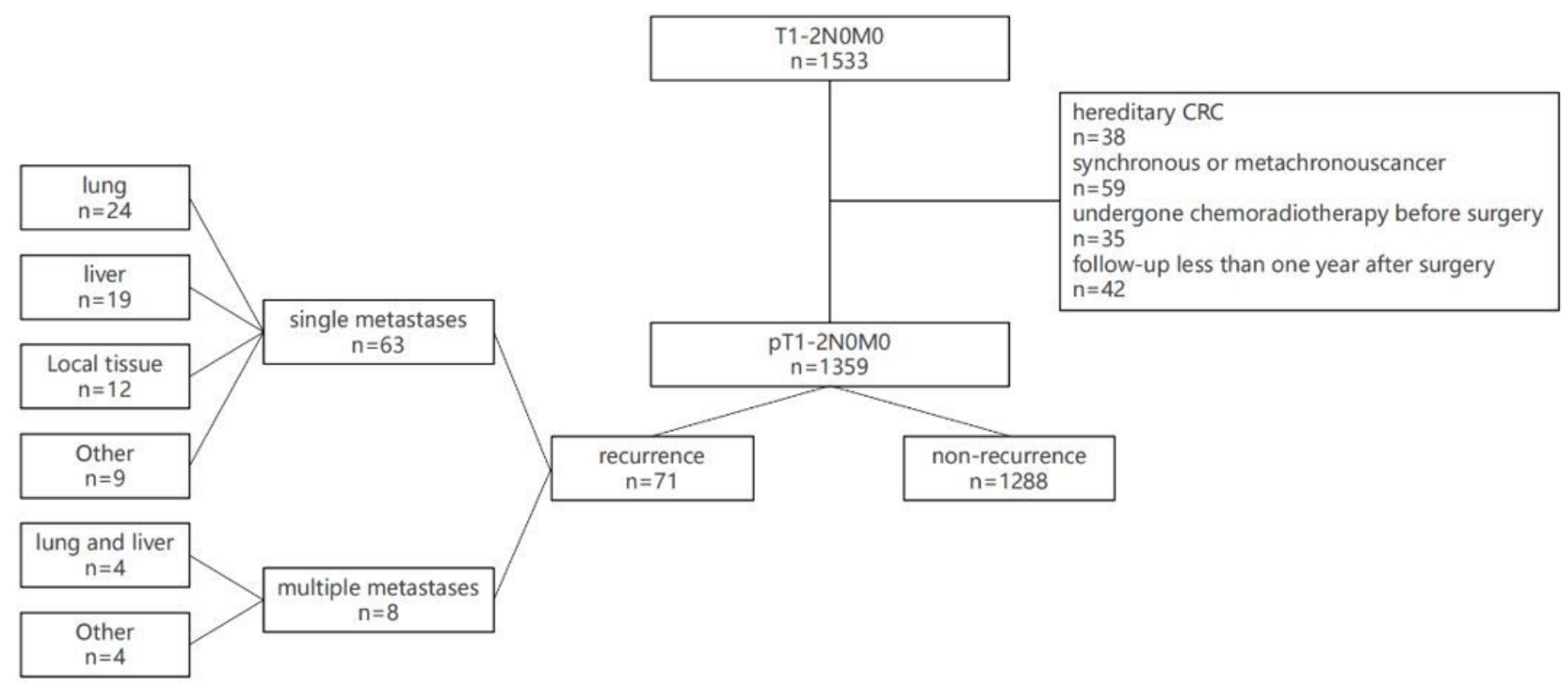

Figure 1

Flow chart of all patients included in the study 


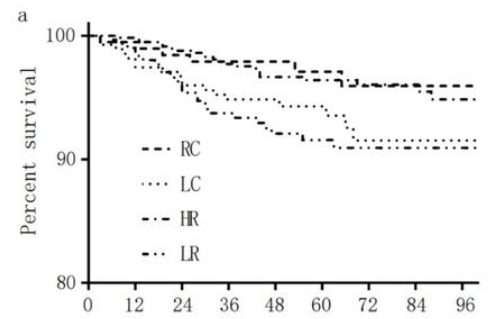

number at risks Time after surgery (month)

$\begin{array}{llllllllll}\text { RC } & 192 & 191 & 189 & 176 & 138 & 101 & 68 & 41 & 28\end{array}$

$\begin{array}{llllllllll}\text { LC } & 274 & 269 & 264 & 239 & 173 & 135 & 78 & 49 & 28\end{array}$

$\begin{array}{llllllllll}\text { HR } & 587 & 586 & 565 & 508 & 416 & 338 & 240 & 173 & 171\end{array}$

$\begin{array}{llllllllll}\text { LR } & 306 & 303 & 290 & 253 & 204 & 162 & 71 & 71 & 41\end{array}$

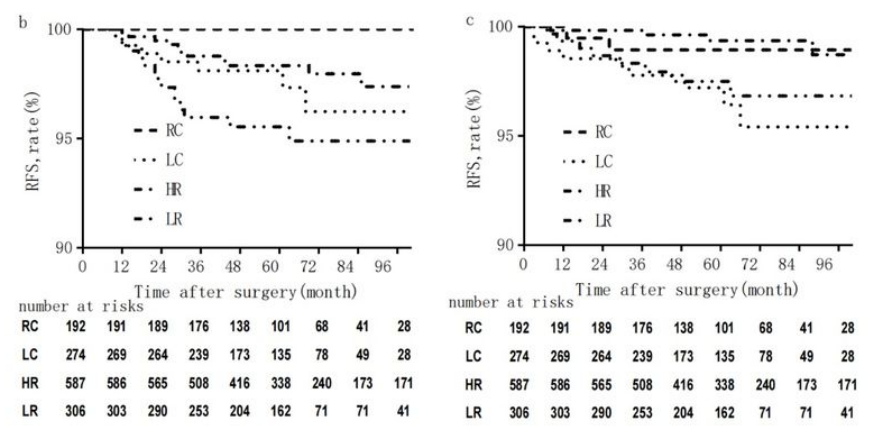

\section{Figure 2}

Recurrence-free survival (RFS) of patients with stage I CRC as a function of a tumor location, b lung metastases,c liver metastases.RC right colon cancer,LC left colon cancer,HR middle-high rectal cancer,LR lower rectal cancer
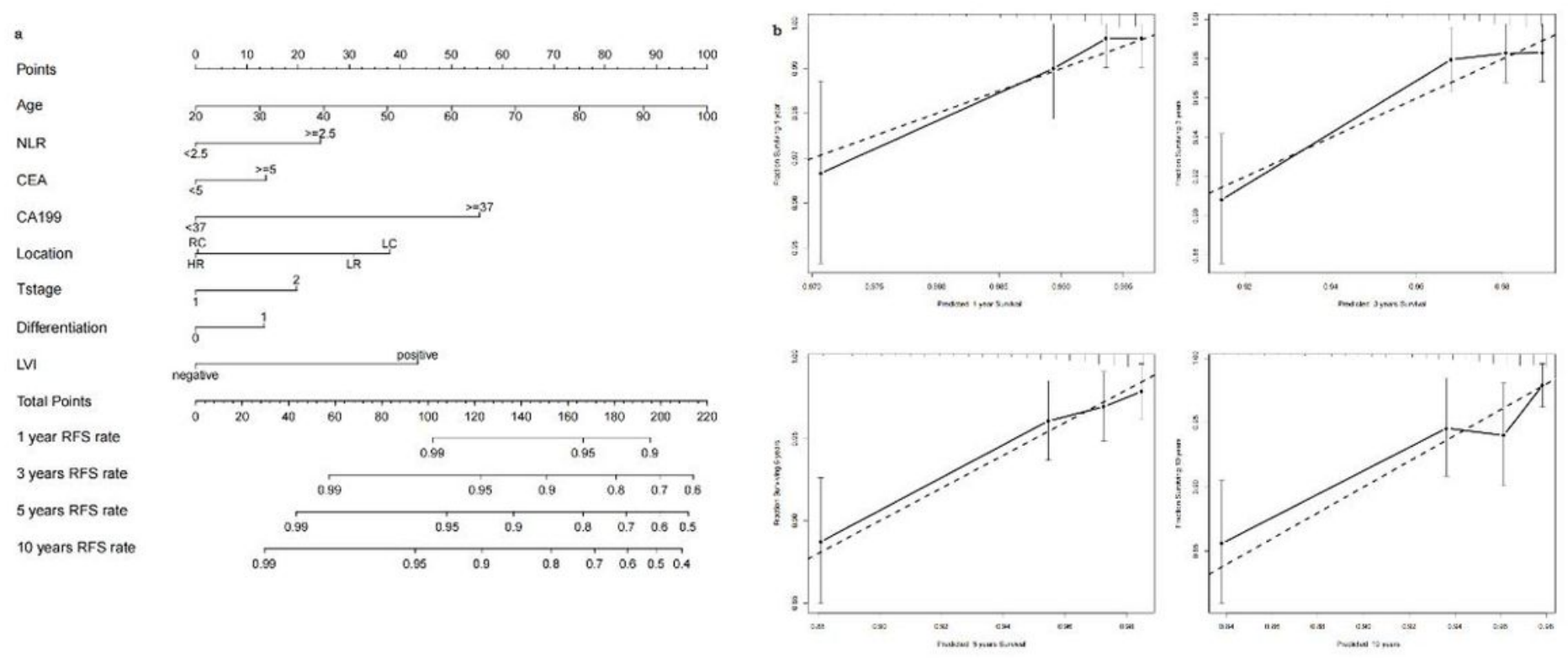

Figure 3 
Predictive Nomogram for Recurrence in Stage I Colorectal Cancer. A Nomogram for Prediction of Recurrence. B Calibration Plot for Nomogram Prediction: Ideal Nomogram (Dashed Line) and Current Nomogram (Solid Line). Vertical Bars Indicate 95\% Confidence Intervals Based on Bootstrap Analysis

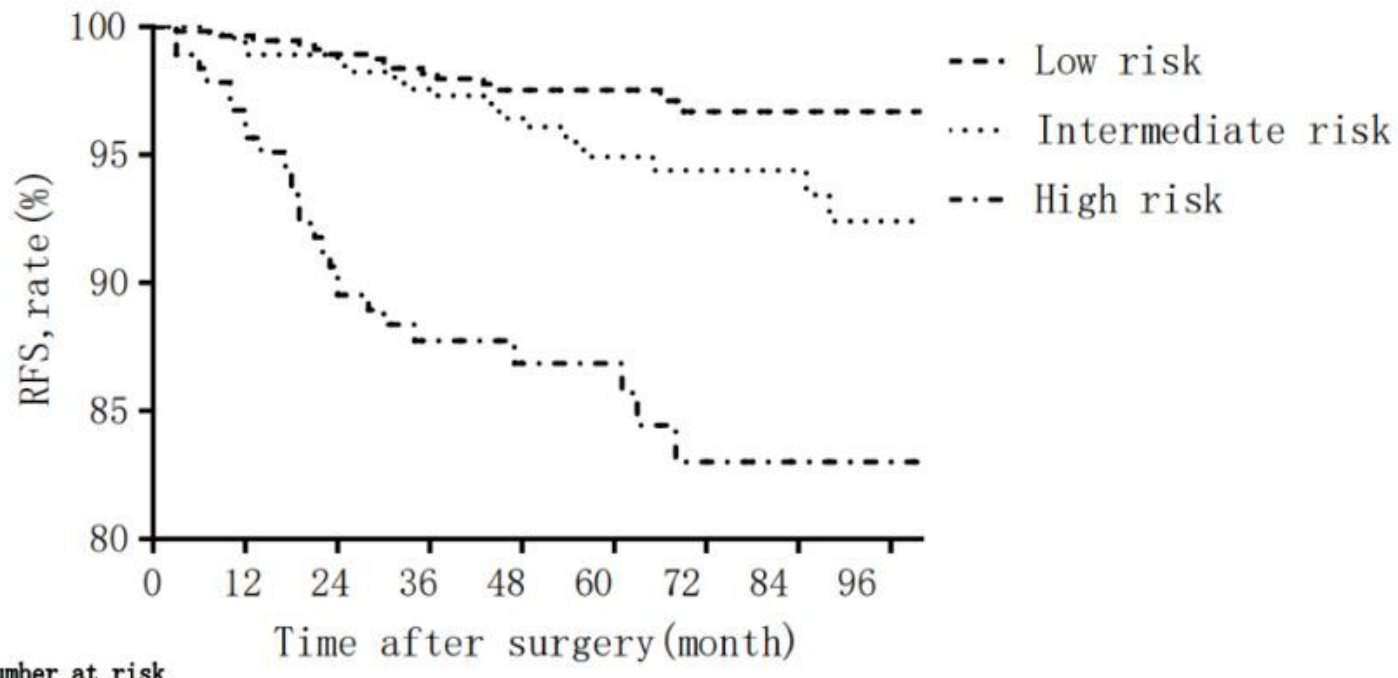

\begin{tabular}{cccccccccc}
\multicolumn{2}{c}{ Number at risk } & & & & & & & \\
Low & 652 & 651 & 636 & 588 & 485 & 382 & 258 & 174 & 116 \\
Inter & 510 & 508 & 490 & 440 & 340 & 254 & 178 & 115 & 72 \\
High & 197 & 191 & 169 & 141 & 106 & 90 & 60 & 42 & 26
\end{tabular}

\section{Figure 4}

RFS curves stratified by the score calculated by the nomogram and was stratified according to the risk score as follows: $<90$, low risk; $100-130$, intermediate risk; and $>130$, high risk. 